\title{
The usability evaluation indicators for IS prototype development process
}

\author{
Mei Luo ${ }^{1}$, Jianhong Jiang ${ }^{2}$ \\ ${ }^{1}$ College of Management, Guilin University of Technology \\ ${ }^{2}$ College of Business, Guilin University of Electronic Technology
}

Keywords: usability; information system; prototype; indicators

\begin{abstract}
At present, most of the researches are focused on the IS usability in company users. This paper discusses the government IS prototype development process evaluation. To analyses whether the IS function performance and effectiveness could achieve the anticipated goal, firstly, summarized the usability indicators from the previous research, constructed the preselected indicators set. Secondly, the Delphi method is adopted to select indicators which users pay more attention to from indicators set. Finally, the indicators system has been constructed by fitting the coordinate coefficient. In order to effectively and widely use IS, the evaluation system can improve the system usability and provide an effective communication between users and developers.
\end{abstract}

\section{Introduction}

Information industry has become the most active, viable and promising pillar industry at information society. Information systems (IS) have become the critical factor affecting business effectiveness and government efficiency. System evaluation is one of the difficulties in the field of IS research. To analyse whether the IS function performance and effectiveness could achieve the anticipated goal, evaluation is necessary [7]. Such evaluations are generally in the information system development is completed after a period of time, those are summative evaluations. Meanwhile in practical applications, especially in the government departments, although the original method or system is inefficient, users do not support new IS which have more complete interface and functions. This phenomenon is more pronounced in the new system to replace the old one. The most important reasons why new IS hard to expand is that users have accustomed to the old method or old IS. The existence of these problems, which led to the new IS did not match the goal that improve efficiency and the level of information. Therefore both development methods and assessment methods of IS need to improve from developing to implying.

Because IS evaluation involving the extensive evaluation content and uncertainties, the actual IS evaluation is very complex [13]. Therefore, the establishment of the IS development process evaluation system can dynamically evaluate IS, timely response to user demand in the face of changes in the external environment and user needs, help developers rapidly develop systems and reduce losses.

The remainder of this paper is divided into two sections. Section 2 describes the prototype development method and usability evaluation. Section 3 describes the usability indicators selection process and method.

\section{The usability evaluation for prototype system}

System usability is the important factor in system adoption and continuous use, the Perceived ease-of-use impact on the behavior intention in Technology Acceptance Model, and the availability of user satisfaction impact on individuals and organizations IS success model, can explain the significance. The research of this paper is process measurement the IS prototype. Following structured method, prototype [8] quickly render the IS development results to manager for avoiding stack and facilitating communication with manager. It is not necessary to have clear goals in early development stage, while gradually approaching the target. Prototype has the features which are short development cycle, low cost, easy to assessment, easy to modify. When the techniques that be used in 
the new product development have been defined, the main risk is uncertain demand, then using evolutionary prototype is more effective than discarded prototype [11]

The basic of implementing process evaluation and improvement is process measurement [14]. Process evaluation is based on a series of standard to evaluate, improve and optimize the software process. By process measurement, the status of software process can be cognized, so that reasonable control the project which is likely to be or has been out of control, and evaluate the effect of the evaluation the process improvements. Using the feedback information obtained from process, the process improvements can find the problems and deficiencies of the current software process, put forward suggestions for improvement, and improve and perfect the software process.

In order to guarantee the product usability, user-cantered design is the most commonly used method [12], the content of this method is that invite users to assess the product and prototype which is in the design or have been released. By analyzing the evaluation data, iterative design the product or prototype until achieving the usability targets 9 Hu Xiaoqin 2009).

The purpose of usability evaluation is to ensure the user-cantered design principles, to test whether the system usability has reached the user's requirements. There are three usability evaluation methods, heuristic method, the method based on data that user use the system, and the method based on quantitative indicators. Among three methods, heuristic evaluation requires the user to take time to participate in the evaluation process of the system in order to identify problems, and requires a relatively long time to evaluate. The second method, data collection takes time to accumulate for getting complete user data, reducing the efficiency of development, more suitable apply in the more mature system development stage. Quantitative indicators method, the research focuses on determining the evaluation indicators and evaluation model, based on this method, the evaluation time is short, user feedback is easy, and suitable for rapid evaluation. By comparing the characteristics of each methods and prototype development process requires a quick feedback, so this paper uses quantitative indicators evaluation method, through the establishment of the indicators to evaluate the usability of the prototype system.

Usability evaluation, experience shows that only up to $20 \%-25 \%$ of the usability problems can be found by the developer, even the most experienced systems developers cannot find all the usability problems [10]. Compared to other IS, government IS has clearer system users. As the active parties of system development process, users participate in the system prototype evaluation, describe, measure and analyse system usability together with the developers. Developers improve the system prototype based on the evaluation results until the usability to user's requirements.

\section{The usability evaluation indicators}

Various definitions of usability are due to scholars' explanations from different angles on usability; these also led to no definite usability indicators to measure the usability [1]). For end users, the usability is crucial, because it measures the user's performance, satisfaction, and productivity, allowing users to be more efficient in carrying out the intended tasks. For managers, the usability is an important determinant that determines the choice of product; therefore it has a direct impact of organizational productivity and performance. For developers, the usability is internal attributes that affect user performance and productivity. This paper is to establish the user-oriented usable evaluation system for dynamic evaluation of the prototype of the government IS.

In order to evaluate the usability of the system, it is necessary to establish a reasonable evaluation indicator system. There are many researches of usability rating scale, but for the of a variety of usability indicators are not the same, because the scholars in the usability field have different view point of the usability indicators, these different scales within the scope of the evaluation is widespread adoption and independent of each other. The scale due to the different definitions of usability and use of different objects which led to different content, these differences include [2]: different indicator name, meaning the same indicators may use a different name; different scales, usability indicator can be combined in different ways by scholars. Current general study of usability rating scale has the following categories. 
Nielsen considered the usability is an aspect that impact system's acceptance, the actual acceptability and social acceptability of the system is different, the usability and effectiveness evaluate the usefulness of the system with evaluate the ability of users to complete a set of tasks. ISO 9241-11 has a clear definition about the usability, it is defined content-oriented view point of usability by the system performance (effectiveness, efficiency) and user (satisfaction). Microsoft also announced the usability guidelines (Microsoft Usability Guidelines, MUG). Not all indicators in usability assessment scale that MUG provided are equally important, but closely related functions of the system, the user's role, the user's purpose and other factors. The properties of usability dominate over the actual system changes [9]. There are many researches about comprehensive evaluation of IS, information system's performance, economic, and degree of completion are often selected as evaluation indicator.

When evaluating usability of different types of systems and different objects, indicator's means and sub-indicator's compositions are different. Some of these indicators in the evaluation system are not a specific object type, and some are more suitable for government portal or website. The research of government IS in this paper, has different objects and actions, so select the appropriate evaluation and screen suitable indicators is necessary to achieve goal that implement a reasonable and accurate evaluation of the system development prototype.

Table 1. Preselect indicators set

\begin{tabular}{|c|c|c|}
\hline Items & Description & Indicators \\
\hline Operability & $\begin{array}{l}\text { The ability that user can use system } \\
\text { effectively. }\end{array}$ & $\begin{array}{l}\text { Convenience navigationV1 } \\
\text { Input modeV2 } \\
\text { Number of inputV3 } \\
\text { Error handlingV4 } \\
\text { The convenience of the input } \\
\text { information to modifyV5 } \\
\text { Jump page modeV6 } \\
\text { Number of pagesV7 }\end{array}$ \\
\hline responsiveness & $\begin{array}{l}\text { System operational efficiency, it is } \\
\text { possible to reduce user's waiting time. }\end{array}$ & $\begin{array}{l}\text { Program start and end timesV8 } \\
\text { Input response timeV9 } \\
\text { Page load timeV10 } \\
\text { Retrieval response timeV11 }\end{array}$ \\
\hline Learnability & $\begin{array}{l}\text { The user-friendliness of system, user } \\
\text { can easily enter the working state. }\end{array}$ & $\begin{array}{l}\text { Reasonable Page navigation V12 } \\
\text { User manual clearV13 } \\
\text { Reasonable page operating tipsV14 } \\
\text { Complete help functionV15 } \\
\text { Useful error messageV16 }\end{array}$ \\
\hline Legibility & $\begin{array}{l}\text { The interface design of system is } \\
\text { reasonable. }\end{array}$ & $\begin{array}{l}\text { Reasonable arrangement of the } \\
\text { elementsV17 } \\
\text { Color V18 } \\
\text { Font sizeV19 } \\
\text { Interpretation of the } \\
\text { informationV20 } \\
\text { Page style unityV21 } \\
\text { Highlight important } \\
\text { informationV22 } \\
\text { Distinguish different types of } \\
\text { informationV23 } \\
\text { Location and format of input } \\
\text { informationV24 }\end{array}$ \\
\hline
\end{tabular}

The principles of establishing the usability evaluation of information system is as blow:

(1) All indicators can be quantified; it is possible to obtain a detailed assessment data based on indicators.

(2) Data can be obtained by measurement and observe, and can be reproduced.

(3) It should have good construct validity.

(4) Indicator can be carried out in accordance with the periodic review, then verify the prototype system's improvement after evaluated.

(5) Evaluation indicator can reflect the actual tasks and operating environment of the user. 
According to the foregoing indicators set, usability indicator set was established in accordance with above principles. Through communication with the government IS users, summarized several usability rating scale previously mentioned, and with reference to specific applicability, the indicators can be attributed to operability, responsiveness, learning and legibility of four aspects, such as Table 1.

To test whether these indicators can truly reflect the users of the system usability evaluation study, the Delphi method was used to make further analysis for usability indicators. Delphi method, also known as expert investigation[6] , is a way that the problem which required to solve were sent to each expert alone for advice through the communication, then recovered and sort out a summary of all expert advice.

Subsequently consolidated comments and questions are feed backed to the experts and then consulted again. Experts modify their original opinions based on comprehensive advice, and then aggregated. So repeatedly, decision making method can gradually made more consistent approach to predictions [4]

Respondent were chosen according to system using time, frequency and proficiency and other options. They are facing with the system and using information system in their daily work frequently. These people belong to user's system users, and their evaluation can represent the user's opinion of system usability. The advice is from Delphi experts that no other persons affected, it is necessary for the evaluation of usability. Especially in the learnability of usability evaluation, due to differences in computer skills, and user which computer skills in a high level were able to easily operate the system, and user has inexperienced computer skills may require high learnability of system. So using the Delphi method allows these evaluators are not affected. Through a unified expert advice, it can ensure the selection of indicators reasonable.

First, design a questionnaire to determine the suitability of indicators. The degree of indicators' importance was divided into five levels based on the Likert method: very important (5 points), important (4 points), generally important (3 points), less important ( 2 points), unimportant (1 point).

Assuming the number of experts is $m$, the number of indicators is $n$ and a few key indicators' computing formulas are available.

Expert's Positive coefficient is $\mathrm{P}$, where $\mathrm{N}$ is the number of questionnaires:

$P=\sum_{i=1}^{m} n_{i} / N$

Concentration degree of expert's advice is $\mathrm{Kj}$, where $\mathrm{kj}$ is maximum score of expert given to the indicator j:

$$
K_{J}=\frac{k_{j}}{m}
$$

Variation coefficient of Expert's advice is $\mathrm{Vj}$, where $\mathrm{Mj}$ is average of indicator $\mathrm{j}$ :

$$
V_{j}=\frac{\sqrt{\frac{1}{m-1} \sum_{i=1}^{m}\left(C_{i j}-M_{j}\right)^{2}}}{M_{j}}
$$

Supose Rij is the ith expert's evaluation grade of jth indicator, the summary grade is Si:

$$
\begin{aligned}
& S_{i}=\sum_{i=1}^{m} R_{i j} \\
& \overline{S_{t}}=\frac{1}{n} \sum_{i=1}^{n} S_{i} \\
& T_{i}=\sum_{i=1}^{m_{i}}\left(n_{i j}^{3}-n_{i j}\right)
\end{aligned}
$$

As shown in Eq.6, where $m$ is the ith evaluation results of the evaluation level evaluator number of repetitions, nij is the evaluation results in the ith evaluator evaluation level of the jth repeat the same number of classes. For the assessment results without the same level of evaluators, $\mathrm{Ti}=0$, so the only assessment results for the same evaluation level of evaluators computing Ti. Expert advice coordination coefficient $\mathrm{W}$ is calculated as:

$$
W=\frac{12 \sum_{i-1}^{n}\left(S_{i}-\overline{S_{i}}\right)^{2}}{m^{2}\left(n^{3}-n\right)-m \sum_{i-1}^{m} T_{i}}
$$


Coordination coefficient represents the difference of evaluation opinion which evaluators given to each indicators. Evaluation expert advice coordination reaction coefficient between each other for each indicator is given considerable differences. $\mathrm{W}$ values between 0 and 1 , $\mathrm{W}$ closer to 1 , indicating that the better degree of coordination.

As shown in Table 2, through three round surveys, $\mathrm{W}$ increased and the value close to 1 . After repeated feedback from evaluator's assessment, evaluators have a more consistent view of the importance of indicators. The results are credible through $\chi^{2}$ significant test.

Table 2. Three round evaluations

\begin{tabular}{cccc}
\hline Round & Number of indicators & W & P \\
\hline 1 & 24 & 0.62 & $<0.05$ \\
2 & 24 & 0.70 & $<0.0001$ \\
3 & 24 & 0.81 & $<0.0001$ \\
\hline
\end{tabular}

As shown in Fig. 1, after three round evaluation surveys, evaluators 'opinions tend to unity. V decreases and remains stable. $\mathrm{K}$ increased slightly, indicating that the views of evaluators have basically been identified. According to the final evaluation results, there are six indicators are considered unimportant for usability evaluation.

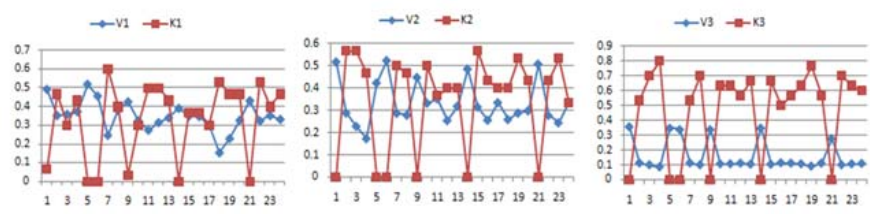

Figure 1. Comparative evaluation coefficient

Table 3. Final usability evaluation indicators

\begin{tabular}{|c|c|c|c|}
\hline Items & Indicators & Items & Indicators \\
\hline \multirow{4}{*}{ Operability } & Input modeV2 & \multirow{4}{*}{ Learnability } & \\
\hline & Number of inputV3 & & User manual clearV13 \\
\hline & Error handlingV4 & & Complete help functionV15 \\
\hline & $\begin{array}{l}\text { The convenience of the input } \\
\text { information to modifyV5 }\end{array}$ & & Useful error messageV16 \\
\hline \multirow{8}{*}{ responsiveness } & \multirow{8}{*}{$\begin{array}{c}\text { Program start and end } \\
\text { timesV8 } \\
\text { Input response timeV9 } \\
\text { Page load timeV10 } \\
\text { Retrieval response timeV11 }\end{array}$} & \multirow{8}{*}{ Legibility } & Reasonable arrangement of the \\
\hline & & & $\begin{array}{l}\text { elementsv1/ } \\
\text { Color V18 }\end{array}$ \\
\hline & & & Font sizeV19 \\
\hline & & & Interpretation of the \\
\hline & & & information V 20 \\
\hline & & & $\begin{array}{l}\text { Highlight important } \\
\text { informationV22 }\end{array}$ \\
\hline & & & Distinguish different types of \\
\hline & & & $\begin{array}{l}\text { informationV23 } \\
\text { Location and format of input } \\
\text { informationV24 }\end{array}$ \\
\hline
\end{tabular}

Based on the feedback from the questionnaire and the actual system usage, construct the evaluation system consist of four aspects, operability, responsiveness, learnability and legibility, which extracted from the system performance and user satisfaction indicators. The final usability is shown in Table 3, compare to the indicators before selection, in operability section, convenience navigation, jump page mode, and number of pages have been removed, users pay more attention on the input operation. In responsiveness section, all indicators have been kept, that indicate users need the system to react fast. In learnability section, reasonable page operating tips and useful error message have been moved, that means users are more likely to learn the system usage method from manual. In legibility section, page style unity and location and format of input information have been moved, that means compared to the details, users pay more attention to the obvious information. 


\section{Conclusions}

Many information systems are hard to effective use after deployment, because users have the different about staff computer skills, operation management gaps and use habit. It's lack of an effective communication way to connect human and system and improve adaptability. This situation may reduce the user's efficiency and increase waste of system resources. This paper established the IS development process evaluation system can dynamically evaluate IS, timely response to user demand in the face of changes in the external environment and user needs, help developers rapidly develop systems and reduce losses.

\section{Acknowledgments}

This research was supported by the Scientific Research Foundation of Guilin University of Technology (Grant No.002401003439) and the Guangxi Natural Science Foundation (Grant No. 2014GXNSFCBA118287), all support is gratefully acknowledged.

\section{References}

[1] Cheng Shiwei, Yuanwu Shi and Shouqian Sun, 2009, Act electronic silica, 37(4A), p.146-150, In Chinese.

[2] Folmer E., Bosch, J. 2004, the journal of systems and software, 70(1-2), p.61-78.

[3] H Linstone, M Turoff, 1975, Delphi Method: Techniques and Applications, First Edition. Addison Wesley.

[4] Hu Xiaoqin and Jianyong Zhang. 2009, New technology of library and information service, 2, p.46-50, In Chinese.

[5] ISO 9241-11, 1994, Ergonomic Requirements for Office Work with Visual Display Terminals (VDTs)-Part II: Guidance on Usability.

[6] Jun Tian, Pengzhu Zhang, Kanliang Wang and Yingluo Wang, 2004, Systems engineering theory practice, 24(1), p.57-62, In Chinese.

[7] Li Enke, Yuxiang Ma, and Guohua Xu, 2000, Journal of the china society for scientific and technical information, 19(2), pp.181-186. In Chinese

[8] Liu Lanjuan and Zhu Yuguang, 2002, Information system analysis and design, publishing house of electronics industry: Beijing. In Chinese.

[9] Lecerof A and Paterno F, 1998, IEEE Transactions on Software Engineering, 24(10), p.863-887.

[10] Nielsen, J, R Molich. 1990, Heuristic Evaluation of User Interfaces, CHI'SO proceedings, SIGCHI Bulletin, Special issue, p.249-256.

[11] Tian Jie, Jianxin Lv and Feng Xu, 2007, Microcomputer Information, 23(24), pp. 13-15, In Chinese.

[12] Vredenburg K, Isensee S, Righi C, 2001, User-Centered Design: An Integrated Approach, Prentice Hall.

[13]Zhang Cuiling, 2006, Information Research, 8, p.61-63.In Chinese

[14]Zhao Qian, Xuan Zhang, Lixia Wang, Cong Liu and Shuai Yang, 2013, Application research of computers, 30(9), p.2730-2739, In Chinese. 